\title{
Estimativas de parâmetros genéticos para a seleção precoce em clones de Eucalyptus spp.
}

\author{
Estimate of genetic parameters for early \\ selection in clones of Eucalyptus spp.
}

\author{
Evandro Vagner Tambarussi' ${ }^{1}$, Bruno Marco de Lima², Rafael da Costa Queiroz ${ }^{3}$, \\ Fabiana Schmidt Bandeira Peres ${ }^{1}$, Donizete da Costa Dias ${ }^{4}$, \\ Maximiliano Kawahata Pagliarini ${ }^{5}$, Fernanda Bortolanza Pereira ${ }^{6}$, \\ João Ricardo Bachega Feijó Rosa ${ }^{7}$ e Gabriel Dehon Sampaio Peçanha Rezende ${ }^{8}$
}

\begin{abstract}
Resumo
O presente trabalho objetivou estimar os parâmetros genéticos e a correlação entre idades juvenil vs adulta em clones de Eucalyptus spp. Os caracteres de interesse econômico como o diâmetro à altura do peito (DAP) e altura (ALT) foram medidos aos três e cinco anos de idade. Os ensaios foram implantados em blocos ao acaso, e cada experimento representa um teste clonal desbalanceado para número de indivíduos e blocos. A herdabilidade no sentido amplo $\left(h_{g}^{2}\right)$ foi baixa para os caracteres avaliados, variou entre 0,1 a $0,26 \%$. A magnitude da herdabilidade média $\left(h_{m}^{2}\right)$ para os testes 4,5 e 7 , variando de 0,27 a 0,95 e 0,31 a 0,95 para DAP e altura, respectivamente. Os ensaios também resultaram altas correlações fenotípicas entre os caracteres nas diferentes idades, com valores variando 0,79 a 0,97 para DAP, como também valores altos de correlações genéticas, variando de 0,53 a 1,00 para os caracteres avaliados. Os resultados obtidos são de fundamental importância para programas de melhoramento genético para as espécies estudadas e para o entendimento das correlações nas idades juvenil vs adulta.
\end{abstract}

Palavras-chave: Melhoramento Florestal; Parâmetros genéticos; Correlações entre idades.

\begin{abstract}
This study aimed to estimate the genetic parameters and correlation between early vs. adult age in Eucalyptus spp. clones. Traits of economic interest as diameter at breast height (DBH) and height (ALT) were measured at three and five years of age. The trials were planted in randomized block design, where each experiment represented an unbalanced clonal test for number of individuals and blocks. The heritability in broad sense $\left(h_{\sigma}^{2}\right)$ was low for the evaluated traits, ranging from 0.1 to $26 \%$. However, the mean heritability $\left(h_{m}^{2}\right)$ was high for tests 4,5 and 7 , varying from 0.27 to 0.95 and 0.31 to 0.95 for $\mathrm{DBH}$ and height, respectively. The tests also showed high phenotypic correlations between traits at different ages, with values ranging from 0.79 to 0.97 for $\mathrm{DBH}$, as well as high values of genetic correlations, ranging from 0.53 to 1.00 . These results have fundamental importance for breeding programs for these species and to understand the correlations of early vs. adult ages.
\end{abstract}

Keywords: Forest Tree Improvement; Genetic parameters; Age-age correlation.

${ }^{1}$ Professor(a) Adjunto(a) do Departamento de Engenharia Florestal. UNICENTRO - Universidade Estadual do Centro-Oeste. PR 153, Km 07 - Riozinho - 84.500-000 - Irati, PR, Brasil. E-mail: evandro.tambarussi@pq.cnpq.br; bandeira.fabiana@gmail.com

${ }^{2}$ Pesquisador Doutor. Fibria Celulose. Rod. BR 158, Km 298 - Fazenda Barra do Moeda- 79601970 - Três Lagoas, MS, Brasil. E-mail: bruno.lima@fibria.com.br

${ }^{3}$ Engenheiro Florestal do Departamento de Engenharia Florestal. UNICENTRO - Universidade Estadual do Centro-Oeste. PR 153, Km 07 - Riozinho - 84.500-000 - Irati, PR, Brasil. E-mail: engfrcosta@outlook.com

${ }^{4}$ Coordenador de Melhoramento Florestal. Fibria Celulose. Rod. Gen. Euryale de Jesus Zerbine - km 84 - $12340-010$ Jacareí, SP, Brasil. E-mail: donizete.dias@fibria.com.br

${ }^{5}$ Doutor em Agronomia. UFGD - Universidade Federal da Grande Dourados. Fazenda Experimental de Ciências Agrárias. Chacara Trinta e Oito - 79800-006 - Dourados , MS, Brasil. E-mail: mpagliarini@ufgd.edu.br

${ }^{6}$ Doutoranda em Ciência Florestal. UNESP - Universidade Estadual Paulista "Júlio de Mesquita Filho" /FCA - Faculdade de Ciências Agronômicas. Rua Doutor José Barbosa de Barros - 18610307 - Botucatu, SP, Brasil. E-mail: fbp.engflorestal@gmail.com

${ }^{7}$ Pós-Doutorando do Departamento de Genética. USP - Universidade de São Paulo / ESALQ - Escola Superior de Agricultura "Luiz de Queiroz". Av. Pádua Dias, 11, Caixa Postal 9 - 13.418-090 - Piracicaba, SP, Brasil. E-mail: jrbachega@gmail. com

${ }^{8}$ Gerente Geral de Tecnologia e Inovação Florestal. Fibria Celulose. Rod. Gen. Euryale de Jesus Zerbine - km 84 - 12340 010 - Jacareí, SP, Brasil. E-mail: gabriel.rezende@fibria.com.br

Sci. For., Piracicaba, v. 45, n. 115, p. 507-517, set. 2017 DOI: dx.doi.org/10.18671/scifor.v45n115.08 
Queiroz et al. - Estimativas de parâmetros genéticos para a seleção precoce em clones de Eucalyptus spp.

\section{INTRODUÇÃO}

Os eucaliptos pertencem à família Myrtaceae, abrangendo mais de 800 espécies, das quais mais de 30 e seus híbridos possuem valor econômico para as mais diversas áreas (FLORES et al. 2016). De forma geral, os eucaliptos apresentam alta adaptabilidade e estabilidade em ambientes distintos, o que o coloca entre as espécies florestais mais estudadas e cultivadas no mundo (IBÁ, 2015; FLORES et al. 2016). O Brasil apresentou no ano de 2015, 7,8 milhões de hectares de plantios florestais dos quais $72 \%$ corresponderam a áreas cultivadas com Eucalyptus spp., com uma produtividade média igual a $36 \mathrm{~m}^{3} \cdot$ ha $^{-1}$.ano (IBÁ, 2016).

Apesar da necessidade de suprir a demanda de madeira nos padrões das indústrias do setor no menor tempo possível, o melhoramento florestal é uma prática demorada, demandando pesquisas para reduzir o tempo de seleção e gerar maior ganho por unidade de tempo (BELTRAME et al., 2012; MARQUES JÚNIOR, 1995). Como subterfúgio, adota-se o procedimento de seleção precoce, que visa fornecer dados experimentais de forma rápida, fácil e com maior versatilidade frente a mudanças das metas dos programas de melhoramento (DEAN et. al., 2006; KAGEYAMA; VENCOVSKY, 1983). A eficiência da seleção precoce está relacionada à alta correlação genética entre os caracteres avaliados em idades juvenis com a performance apresentada na idade de rotação (FERREIRA, 2005; MORAES et al., 2014).

Dessa forma, o estudo teve como objetivos correlacionar caracteres de interesse comercial nas fases juvenil e adulta em clones de Eucalyptus e estimar os parâmetros genéticos para os materiais genéticos avaliados.

\section{MATERIAL E MÉTODOS}

Os dados utilizados, cedidos pela Empresa Fibria Celulose S.A., são provenientes de sete experimentos clonais com as espécies Eucalyptus urophylla S.T. Blake, Eucalyptus grandis Hill ex Maiden, Eucalyptus saligna Sm. e um experimento composto por um híbrido de Eucalyptus spp. Os ensaios de campo apresentavam idades de três e cinco anos no momento das avaliações do diâmetro à altura do peito (DAP) e da altura comercial (ALT) foram os caracteres avaliados. Os experimentos estão em delineamento em blocos ao acaso com oito plantas/parcela em espaçamento $3 \times 2 \mathrm{~m}$, instalados em duas cidades do interior do Estado de São Paulo (Jacareí e Capão Bonito). A região de Jacareí apresenta pluviosidade média de $1.301 \mathrm{~mm}$ e altitude de $567 \mathrm{~m}$, enquanto Capão Bonito encontra-se a $728 \mathrm{~m}$ de altitude com pluviosidade média de $1.628 \mathrm{~mm}$ (Tabela 1).

Tabela 1. Caracterização dos experimentos avaliados.

Table 1. Evaluated trials characterization.

\begin{tabular}{|c|c|c|c|c|c|c|}
\hline Local & Solo & Experimento & Espécie & $\mathrm{N}^{\circ}$ Clones & $\mathrm{N}^{\circ}$ Blocos & Fazenda \\
\hline \multirow{4}{*}{$\begin{array}{l}\text { Jacareí/SP } \\
\left(23^{\circ} 18^{\prime} 19^{\prime \prime S} ; 45^{\circ} 57^{\prime} 57^{\prime \prime} \mathrm{W}\right)\end{array}$} & LV2 & $1(2002)$ & E. urophylla & 45 & 6 & Gaspar \\
\hline & PV1 & $2(2002)$ & E. grandis & 22 & 6 & Baronesa \\
\hline & PV1 & $3(2003)$ & E. grandis & 41 & 6 & Sitio Velho \\
\hline & PV1 & $4(2006)$ & Híbrido & 31 & 30 & São Joaquim \\
\hline \multirow{3}{*}{$\begin{array}{l}\text { Capão Bonito/SP } \\
\left(24^{\circ} 00^{\prime} 21^{\prime \prime S} ; 48^{\circ} 20^{\prime} 58^{\prime \prime} \mathrm{W}\right)\end{array}$} & LVA1 & $5(2001)$ & E. grandis & 40 & 6 & Boa Vista \\
\hline & LVA1 & $6(2001)$ & E. grandis & 58 & 6 & Capão Alto \\
\hline & LVA1 & 7 (2005) & E. saligna & 63 & 6 & Olho D'Água \\
\hline
\end{tabular}

LV2: Associação Latossolo Vermelho + Neossolo Regolítico; PVI: Argissolo Vermelho-Amarelo eutrófico; LVAI: Latossolo Vermelho álico; LV2: Association Rhodic + Entisol; PVI: Ultisol eutrophic; LVAI: Red Alic Latosol.

Os valores genotípicos de cada caráter analisado foram estimados por meio do software Selegen-REML/BLUP (RESENDE, 2002a), a partir do procedimento da Máxima Verossimilhança Restrita (REML, Restricted Maximum Likelihood) proposto por Patterson e Thompson (1971), empregando-se o modelo linear misto para avaliação de clones não aparentados, em blocos ao acaso, com várias árvores por parcela, conforme segue:

$$
\mathrm{Y}=\mathrm{Xb}+\mathrm{Zg}+\mathrm{Wp}+\mathrm{e}
$$

em que $Y$ é o vetor de observações fenotípicas para um dado caráter; $X$ é a matriz de incidência 
para os efeitos fixos; $\boldsymbol{b}$ é o vetor dos efeitos fixos (média geral e efeitos de bloco); $Z$ é a matriz de incidência para os efeitos aleatórios genotípicos; $g$ é o vetor dos efeitos aleatórios genotípicos; $W$ é a matriz de incidência dos efeitos da parcela; $\boldsymbol{p}$ é o vetor dos efeitos da parcela; e $\boldsymbol{e}$ é o vetor dos erros ou resíduos (aleatórios).

O método REML foi utilizado por meio de algoritmos iterativos EM (do inglês Expectation and Maximization), que realizam estimativas dos efeitos ajustados dos vetores calculados por meio de resoluções de matrizes. Assim, foram calculados os seguintes parâmetros:

a) Variância ambiental entre parcelas $\left(\hat{\sigma}_{c}^{2}\right): \hat{\sigma}_{c}^{2}=\left[\hat{c}^{\prime} \hat{c}+\hat{\sigma}_{e}^{2} t r^{33}\right] / s_{1}$

b)Variância residual (ambiental+nãoaditiva) $\left(\hat{\sigma}_{e}^{2}\right): \hat{\sigma}_{e}^{2}=\left[y^{\prime} y-\hat{r}^{\prime} X^{\prime} y-\hat{a}^{\prime} Z y-\hat{c}^{\prime} W^{\prime} y\right] /[N-r(x)]$

c) Herdabilidade no sentido amplo $\left(h_{g}^{2}\right): h_{g}^{2}=\frac{\sigma_{g}^{2}}{\sigma_{g}^{2}+\sigma_{p}^{2}+\sigma_{e}^{2}}$

d) Coeficiente dos efeitos ambientais entre parcelas $\left(c^{2}\right.$ parc $): c^{2}$ parc $=\frac{\sigma_{p}^{2}}{\sigma_{g}^{2}+\sigma_{p}^{2}+\sigma_{e}^{2}}$

e) Herdabilidade média de clones $\left(h_{m}^{2}\right): h_{m}^{2}=\frac{\sigma_{g}^{2}}{\sigma_{g}^{2}+\frac{\sigma_{c}^{2}}{b}+\frac{\sigma_{e}^{2}}{n b}}$

f) Coeficiente de Variação experimental $\left(C V_{\exp }(\%)\right): C V_{\exp }(\%)=\frac{\sqrt{\frac{\sigma_{\varepsilon}^{2}}{n}+\sigma_{c}^{2}}}{m} .100$

g) Coeficiente de variação genética $\left(C V_{g}(\%)\right): C V_{g}(\%)=\frac{\sqrt{\sigma_{g}^{2}}}{m} .100$

h) Relação entre o coeficiente de variação genético pelo ambiental $\left(C V_{\tau}\right): C V_{r}=\frac{C V_{g}(\%)}{C V_{\exp }(\%)}$

i) Variância Genética $\left(\sigma_{B}^{2}\right): \sigma_{B}^{2}=\sigma_{a}^{2}+\sigma_{D}^{2}+\sigma_{i}^{2}$

A correlação genética $\left(\hat{r}_{g}\right)$ entre dois caracteres foi estimada por

$$
\hat{r}_{g}=\frac{C \hat{O} V_{a(x, y)}}{\hat{\sigma}_{a(x)}^{2} \cdot \hat{\sigma}_{a(y)}^{2}}
$$

em que $C \hat{O} V_{a(x, y)}$ é a covariância genética aditiva entre x e y; $\hat{\sigma}_{a(x)}^{2}$ é o componente de variância genética aditiva para x; e $\hat{\sigma}_{a(y)}^{2}$ é o componente de variância genética aditiva para y.

A correlação fenotípica $\left(r_{f}\right)$ entre dois caracteres foi estimada por

$$
\hat{r}_{f}=\frac{C \hat{O} V_{f(x, y)}}{\sqrt{\hat{\sigma}_{f(x)}^{2} \cdot \hat{\sigma}^{2}}}
$$

em que $C \hat{O} V_{f(x, y)}$ é a covariância fenotípica aditiva entre x e y; $\hat{\sigma}_{f(x)}^{2}$ é o componente de variância genética aditiva para $\mathrm{x}$; e $\hat{\sigma}_{f(y)}^{2}$ é o componente de variância genética aditiva para y. Tais correlações foram obtidas a partir dos componentes de variância estimados pelo método REML/BLUP (máxima verossimilhança restrita/melhor predição linear não viciada), implementado no software Selegen (RESENDE, 2002a). Além disso, as correlações genéticas e fenotípicas entre caracteres foram estimadas a partir do coeficiente de correlação de Pearson e exploradas graficamente, por meio do pacote psych (REVELLE, 2016), disponível no software estatístico R (R CORE TEAM, 2016). Um nível de $1 \%$ de significância foi utilizado para declarar correlação estatisticamente significativa. Ademais, foi possível visualizar graficamente a distribuição dos dados dos caracteres e possíveis tendências de ajustes entre os mesmos.

Por meio do método de Análise de Deviance avaliou-se a significância estatística entre os tratamentos. Para sua composição foi realizado os seguintes passos, conforme Resende (2007): i) obtenção do ponto de máximo do logaritmo da função de verossimilhança residual (LogL) para os modelos com ou sem efeitos a serem testados; ii) cálculo da Deviance ( $D=-2 \log L$ ) para modelos com e sem o efeito a ser testado; iii) obtenção da razão de verossimilhança (LR, do inglês Likelihood Ratio) por meio das diferenças entre as Deviances para modelos sem e com o efeito a ser testado; iv) Teste, via LRT (do inglês Likelihood Ratio Test), da significância da diferença usando o teste qui-quadrado com 1 grau de liberdade $(\mathrm{GL}=1)$ 


\section{RESULTADOS E DISCUSSÃO}

Houve significância dos efeitos genotípicos nas duas idades avaliadas para os caracteres avaliados nos clones pela análise de Deviance $\left(\mathrm{LTR}_{\text {clone }}\right.$ ) para todos os experimentos, exceto para o experimento 3 (Tabela 2), indicando variabilidade genética entre os materiais genéticos em estudo, o que é benéfico a seleção (ZIMBACK et al., 2011).

Tabela 2. Análise de Deviance e teste de razão da verossimilhança (LRT) dos clones e das parcelas para diâmetro à altura do peito (DAP), em cm, e altura (ALT), em m, aos três (DAP3, ALT3) e cinco (DAP3, ALT3) anos após o plantio. Local: Jacareí - SP e Capão Bonito - SP.

Table 2. Deviance analysis and likelihood ratio test (LRT) of clones and plots to diameter at breast height (DAP), in $\mathrm{cm}$, and height (ALT), in $\mathrm{m}$, to plants three (DAP3, ALT3) and five (DAP5, ALT5) years old. Location: Jacareí - SP and Capão Bonito - SP.

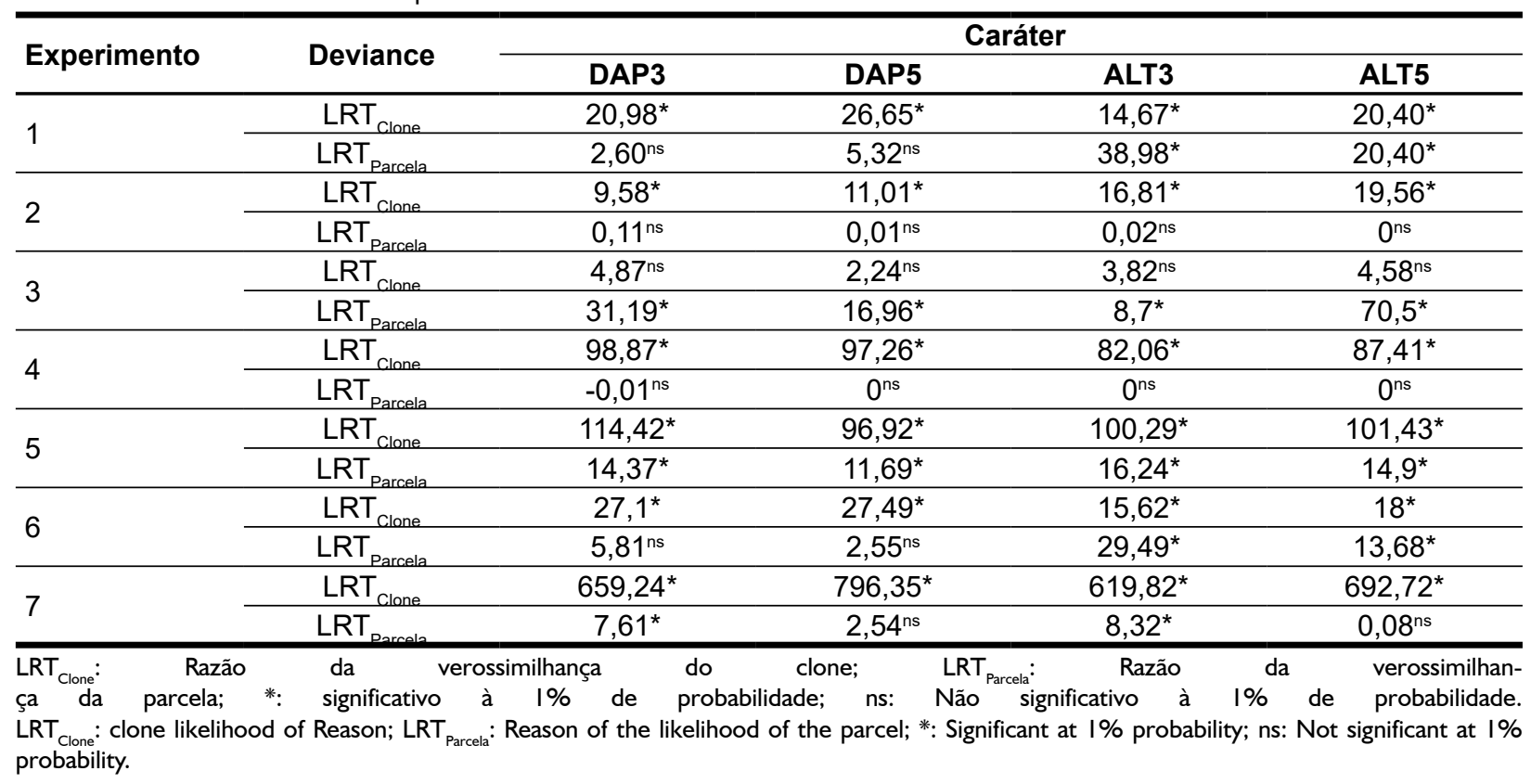

A $\hat{\sigma}_{g}$ para o caráter DAP aos três e cinco anos de idade variou entre 0,09 (experimento 3) a 9,42 (experimento 5), enquanto que para ALT, os valores variavam de 0,08 a 12,12 (Tabela 3), o que indica variação genética entre os caracteres ao decorrer dos anos. No caso de $\sigma_{p a r c}^{2} \mathrm{e} \sigma_{\theta}^{2}$, houve pouca variação para ambos os caracteres aos três e cinco anos (Tabela 3), indicando que as $\sigma_{f}^{2}$ encontradas entre os indivíduos são de caráter genético (GARCIA, 2005).

Os coeficientes $h_{g}^{2}$ foram baixos, variando entre 0,01 (Experimento $3 \mathrm{em}$ todos os caracteres) a 0,26 (DAP5 no Experimento 7), para DAP e ALT (Tabela 4), o que não resulta favorável à escolha de indivíduos com caracteres superiores desejáveis comercialmente, pois quanto menor a herdabilidade de um dado caráter, menor é o seu controle genético (FALCONER; MACKAY, 1996). A $h_{m}^{2}$ apresentou valores superiores aos de $h_{g}^{2}$ e variação com o aumento da idade. Valores altos de $h_{m}^{2}$ são indicados para a seleção de materiais genéticos de interesse. Para DAP, a $h_{m}^{2}$ variou entre 0,27 (DAP5, experimento 3) e 0,98 (DAP5, experimento 4), enquanto para ALT os valores variaram entre 0,31 (ALT5, experimento 3) e 0,97 (ALT3 e ALT5, experimento 4) (Tabela 4). Em trabalhos observados na literatura, os valores de $h_{m}^{2}$ para estes caracteres apresentam-se elevados, superiores a 0,70, em diferentes idades (BELTRAME et al., 2012; MASSARO et al., 2010; MORAES et al.; 2014). Dessa forma, a partir dos resultados obtidos, a seleção de materiais genéticos deve ter por base a herdabilidade média entre clones e não a herdabilidade no sentido amplo.

$\mathrm{O} c_{\text {parc }}^{2}$ mensura a variabilidade dentro dos blocos, logo, quanto mais elevado este valor, maior será a variância ambiental (ROCHA, 2006). Os caracteres avaliados tiveram baixa variância ambiental, pois os valores variaram entre 0,01 (experimento 2 e 7) a 0,09 (experimento 4) para DAP e entre 0,01 (experimento 2 e 7) até 0,10 (experimento 1) para ALT (Tabela 4). 
Tabela 3. Estimativa das variâncias genéticas de parcela, ambiental e fenotípica em clones de Eucalyptus spp. para diâmetro à altura do peito (DAP) e altura (ALT), aos três (DAP3, ALT3) e aos cinco (DAP5, ALT5) anos de idade. Local: Jacareí - SP e Capão Bonito - SP.

Table 3. Estimation of genetic variances (term, environmental and phenotypic) for Eucalyptus spp clones. For the character diameter at breast height (DBH cm) and height ( $\mathrm{m} \mathrm{ALT}$ ) to three (DAP3, ALT3) and five (DAP5, ALT5) years old. Location: Jacareí - SP and Capão Bonito - SP.

\begin{tabular}{|c|c|c|c|c|c|}
\hline \multirow{2}{*}{ Experimento } & \multirow{2}{*}{ Parâmetro } & \multicolumn{4}{|c|}{ Caráter } \\
\hline & & DAP3 & DAP5 & ALT3 & ALT5 \\
\hline \multirow{4}{*}{1} & $\hat{\sigma}_{g}$ & 0,26 & 0,61 & 0,33 & 0,96 \\
\hline & $\sigma_{p a r c}^{2}$ & 0,14 & 0,39 & 0,70 & 1,70 \\
\hline & $\sigma_{e}^{2}$ & 6,30 & 11,12 & 5,94 & 13,48 \\
\hline & $\sigma_{f}^{2}$ & 3,71 & 12,13 & 6,97 & 16,13 \\
\hline \multirow{4}{*}{2} & $\hat{\sigma}_{g}$ & 0,43 & 0,68 & 0,98 & 2,23 \\
\hline & $\sigma_{p a r c}^{2}$ & 0,08 & 0,06 & 0,07 & 0,11 \\
\hline & $\sigma_{t}^{2}$ & 11,10 & 18,43 & 16,62 & 34,88 \\
\hline & $\sigma_{f}^{2}$ & 11,61 & 19,17 & 17,67 & 37,22 \\
\hline \multirow{4}{*}{3} & $\hat{\sigma}_{g}$ & 0,09 & 0,11 & 0,08 & 0,21 \\
\hline & $\sigma_{p a r c}^{2}$ & 0,28 & 0,34 & 0,13 & 1,11 \\
\hline & $\sigma_{\epsilon}^{2}$ & 6,36 & 11,61 & 6,86 & 13,41 \\
\hline & $\sigma_{f}^{2}$ & 0,09 & 0,11 & 0,08 & 0,21 \\
\hline \multirow{4}{*}{4} & $\hat{\sigma}_{g}$ & 1,77 & 3,56 & 1,75 & 5,36 \\
\hline & $\sigma_{p a r c}^{2}$ & 1,07 & 2,00 & 1,10 & 3,28 \\
\hline & $\sigma_{e}^{2}$ & 8,57 & 16,04 & 8,82 & 26,24 \\
\hline & $\sigma_{f}^{2}$ & 11,42 & 21,61 & 11,67 & 34,88 \\
\hline \multirow{4}{*}{5} & $\hat{\sigma}_{g}$ & 6,53 & 9,42 & 5,80 & 12,12 \\
\hline & $\sigma_{p a r c}^{2}$ & 1,47 & 2,34 & 1,58 & 3,14 \\
\hline & $\sigma_{\epsilon}^{2}$ & 26,06 & 44,64 & 23,51 & 49,53 \\
\hline & $\sigma_{f}^{2}$ & 34,07 & 56,40 & 30,90 & 64,80 \\
\hline \multirow{4}{*}{6} & $\hat{\sigma}_{g}$ & 0,55 & 0,97 & 0,41 & 1,26 \\
\hline & $\sigma_{p a r c}^{2}$ & 0,38 & 0,47 & 0,81 & 1,67 \\
\hline & $\sigma_{e}^{2}$ & 14,12 & 26,17 & 11,67 & 35,59 \\
\hline & $\sigma_{f}^{2}$ & 15,05 & 27,60 & 12,89 & 38,53 \\
\hline \multirow{4}{*}{7} & $\hat{\sigma}_{g}$ & 2,61 & 6,00 & 1,94 & 6,34 \\
\hline & $\sigma_{p a r c}^{2}$ & 0,11 & 0,11 & 0,09 & 0,02 \\
\hline & $\sigma_{e}^{2}$ & 9,14 & 17,30 & 6,92 & 20,23 \\
\hline & $\sigma_{f}^{2}$ & 11,87 & 23,41 & 8,95 & 26,60 \\
\hline
\end{tabular}

$\hat{\bar{\sigma}}_{g}$ : Variância genotípica; ${ }_{2} \sigma_{p a r c}^{2}$ : Variância ambiental entre parcelas; ${ }_{2} \sigma_{e}^{2}$ : Variância residual; $\sigma_{e}^{2}$ : Variância fenotípica individual. $\hat{\sigma}_{g}^{g}$ : genotypic variance; $\sigma_{p a r c}^{2}$ : environmental variance between plots; $\sigma_{\theta}^{2}$ : residual variance; $\sigma_{\theta}^{2}$ : individual phenotypic variance. 
Queiroz et al. - Estimativas de parâmetros genéticos para a seleção precoce em clones de Eucalyptus spp.

Tabela 4. Estimativa das herdabilidades e do coeficiente dos efeitos ambientais entre parcelas em clones de Eucalyptus spp. para diâmetro à altura do peito (DAP), em cm, e altura (ALT), em m, aos três (DAP3, ALT3) e cinco (DAP5, ALT5) anos de idade. Local: Jacareí - SP e Capão Bonito - SP.

Table 4. Heritability estimate and the coefficient of environmental effects between plots of Eucalyptus spp. clones. for the character diameter at breast height (DBH) and height (ALT) to three (DAP3, ALT3) and five (DAP5, ALT5) years old. Location: Jacareí - SP and Capão Bonito - SP.

\begin{tabular}{|c|c|c|c|c|c|}
\hline \multirow{2}{*}{ Experimento } & \multirow{2}{*}{ Parâmetros } & \multicolumn{4}{|c|}{ Caráter } \\
\hline & & DAP3 & DAP5 & ALT3 & ALT5 \\
\hline \multirow{3}{*}{1} & $h_{g}^{2}$ & $0,04( \pm 0,01)$ & $0,05( \pm 0,01)$ & $0,05( \pm 0,01)$ & $0,06( \pm 0,01)$ \\
\hline & $c_{p a}^{2^{g}}$ & 0,02 & 0,03 & 0,10 & 0,10 \\
\hline & $h_{m}^{2}$ & 0,63 & 0,67 & 0,58 & 0,63 \\
\hline \multirow{3}{*}{2} & $\overline{h^{2}}$ & $0,04( \pm 0,02)$ & $0,03( \pm 0,02)$ & $0,05( \pm 0,02)$ & $0,06( \pm 0,02)$ \\
\hline & $c_{p a}^{2^{g}}$ & 0,01 & 0,01 & 0,01 & 0,01 \\
\hline & $h_{m}^{2}$ & 0,64 & 0,63 & 0,73 & 0,75 \\
\hline \multirow{3}{*}{3} & $\overline{h_{g}^{2}}$ & $0,01( \pm 0,01)$ & $0,01( \pm 0,01)$ & $0,01( \pm 0,01)$ & $0,01( \pm 0,01)$ \\
\hline & $c_{\text {parc }}^{g}$ & 0,04 & 0,03 & 0,02 & 0,07 \\
\hline & $h_{m}^{2}$ & 0,34 & 0,27 & 0,34 & 0,31 \\
\hline \multirow{3}{*}{4} & $h_{g}^{2}$ & $0,15( \pm 0,04)$ & $0,16( \pm 0,04)$ & $0,15( \pm 0,04)$ & $0,15( \pm 0,07)$ \\
\hline & $c_{\text {parc }}^{2^{8}}$ & 0,09 & 0,09 & 0,09 & 0,09 \\
\hline & $h_{m}^{2}$ & 0,97 & 0,98 & 0,97 & 0,97 \\
\hline \multirow{3}{*}{5} & $\overline{h_{g}^{2}}$ & $0,19( \pm 0,03)$ & $0,17( \pm 0,03)$ & $0,19( \pm 0,03)$ & $0,19( \pm 0,03)$ \\
\hline & $c_{\text {parc }}^{2^{8}}$ & 0,04 & 0,04 & 0,05 & 0,05 \\
\hline & $h_{m}^{2}$ & 0,90 & 0,89 & 0,90 & 0,90 \\
\hline \multirow{3}{*}{6} & $h_{g}^{2}$ & $0,04( \pm 0,01)$ & $0,03( \pm 0,01)$ & $0,03( \pm 0,01)$ & $0,03( \pm 0,01)$ \\
\hline & $c_{p a l}^{2^{\circ}}$ & 0,02 & 0,02 & 0,06 & 0,04 \\
\hline & $h_{m}^{2}$ & 0,65 & 0,65 & 0,56 & 0,59 \\
\hline \multirow{3}{*}{7} & $h_{g}^{2}$ & $0,22( \pm 0,02)$ & $0,26( \pm 0,02)$ & $0,22( \pm 0,02)$ & $0,24( \pm 0,02)$ \\
\hline & $c_{p a l}^{2^{8}}$ & 0,01 & 0,01 & 0,01 & 0,01 \\
\hline & $h_{m}^{2}$ & 0,94 & 0,95 & 0,94 & 0,95 \\
\hline
\end{tabular}

$h_{g}^{2}$ : Herdabilidade individual no sentido amplo; $c_{\text {parc }}^{2}:$ coeficiente dos efeitos ambientais entre parcelas; $h_{m}^{2}:$ Herdabilidade média entre clones. $h_{g}^{2}:$ Individual Heritability in the broad sense; $c_{\text {parc }}^{2}$ : coefficient of environmental effects between plots; $h_{m}^{2}:$ Heritability average between clones.

O $\mathrm{CV}_{t} \%$ variou de 6,2\% (ALT3, experimento 3) a 16,8\% (DAP5, experimento 5) (Tabela 5), o que indica boas condições dos experimentos (PIMENTEL-GOMES; GARCIA, 2002) e corroboram com valores observados em outros trabalhos com Eucalyptus spp. (MASSARO et al., 2010; MORAES et al., 2014; PINTO et al., 2014). Os valores de $C V_{g} \%$ obtidos no trabalho são considerados de baixa magnitude, porém são valores semelhantes aos obtidos por outros autores. Massaro et al. (2010) observaram valores de $C V_{g} \%$ que variaram entre 7,9 e 13,8\% para DAP e 5,8 e 9,6\% para ALT em clones de Eucalyptus spp. aos 2, 4 e 6 anos de idade. Estimativas similares às obtidas neste trabalho já foram observadas por Pinto et al. (2014). Segundo estes mesmos autores, e corroborando com nossos resultados, o $C V_{g} \%$ tende a aumentar com o avanço da idade.

$\mathrm{O} C V_{r}$ resultou em valores maiores que 1 para os experimentos 4, 5 e 7 (Tabela 5). De acordo com Vencovsky (1978), Cruz et al. (2004) e Garcia (2005), altos $C V_{g} \%$ e $C V_{\tau}$ iguais ou superiores a 1 indicam situação favorável à seleção. Os resultados indicam também que os resultados obtidos são condizentes ao esperado em situação de erro experimental reduzido, maximizando a acurácia (RESENDE, 2007).

De acordo com Resende e Duarte (2007), a $r_{a a}$ classifica-se em muito alta $(>0,90)$, alta $(0,70$ a 0,90$)$, moderada $(0,50$ a 0,70$)$ e baixa $(<0,50)$. Para os caracteres avaliados, os valores de $r_{a a}^{*}$ classificam-se como alto a muito alto, variando de 0,76 (ALT3) a 0,99 (experimento 4 em todos caracteres), exceto para o experimento 3, que foram moderados, variando entre 0,52 (DAP5) e 0,59 (DAP3) (Tabela 5). Seguindo o princípio de que quanto maior for a acurácia maior será a precisão da seleção e, portanto, maior será o ganho genético (RESENDE, 1995), dado que uma acurácia foi superior a 50\%, isso indica boa precisão na seleção dos genótipos (RESENDE, 2002b), os dois caracteres aqui avaliados apresentam-se, nesta perspectiva, passíveis para seleção de materiais. 
Tabela 5. Estimativa dos parâmetros genéticos em clones de Eucalyptus spp. para diâmetro à altura do peito (DAP) e altura (ALT) aos três (DAP3, ALT3) e aos cinco (DAP5, ALT5) anos de idade. Local: Jacareí - SP e Capão Bonito - SP.

Table 5. Estimation of genetic parameters for Eucalyptus spp. clones for the character diameter at breast height $(\mathrm{DBH})$ and height (ALT) at three (DAP3, ALT3) and five (DAP5, ALT5) years old. Location: Jacareí - SP and Capão Bonito - SP.

\begin{tabular}{|c|c|c|c|c|c|}
\hline \multirow{2}{*}{ Experimento } & \multirow{2}{*}{ Parâmetros } & \multicolumn{4}{|c|}{ Caráter } \\
\hline & & DAP3 & DAP5 & ALT3 & ALT5 \\
\hline \multirow{6}{*}{1} & $C V_{g}(\%)$ & 4,58 & 6,16 & 3,90 & 5,11 \\
\hline & $C V_{e}(\%)$ & 8,6 & 10,5 & 8,1 & 9,6 \\
\hline & $C V_{r}$ & 0,53 & 0,59 & 0,48 & 0,53 \\
\hline & $r_{a a}$ & 0,79 & 0,82 & 0,76 & 0,79 \\
\hline & $r_{g g d}$ & 0,92 & 0,90 & 0,74 & 0,77 \\
\hline & $\hat{x}$ & 11,23 & 12,72 & 14,74 & 19,15 \\
\hline \multirow{6}{*}{2} & $C V_{g}(\%)$ & 5,62 & 6,02 & 5,83 & 6,51 \\
\hline & $C V_{e}^{\circ}(\%)$ & 10,4 & 11,2 & 8,6 & 9,2 \\
\hline & $C V_{r}$ & 0,54 & 0,54 & 0,67 & 0,71 \\
\hline & $r_{a a}$ & 0,80 & 0,80 & 0,85 & 0,86 \\
\hline & $r_{g g d}$ & 0,97 & 0,98 & 0,99 & 0,99 \\
\hline & $\hat{x}$ & 11,65 & 13,72 & 16,94 & 22,94 \\
\hline \multirow{6}{*}{3} & $C V_{g}(\%)$ & 2,65 & 2,34 & 1,83 & 2,06 \\
\hline & $C V_{e}(\%)$ & 8,9 & 9,4 & 6,2 & 7,5 \\
\hline & $C V_{r}$ & 0,29 & 0,25 & 0,29 & 0,27 \\
\hline & $r_{a a}$ & 0,59 & 0,52 & 0,58 & 0,56 \\
\hline & $r_{g g d}$ & 0,67 & 0,66 & 0,79 & 0,53 \\
\hline & $\hat{x}$ & 11,56 & 14,21 & 15,90 & 22,19 \\
\hline \multirow{6}{*}{4} & $C V_{g}(\%)$ & 10,57 & 12,63 & 7,15 & 10,29 \\
\hline & $C V_{e}(\%)$ & 9,2 & 10,6 & 6,4 & 9,0 \\
\hline & $C V_{r}$ & 1,14 & 1,19 & 1,12 & 1,14 \\
\hline & $r_{a a}$ & 0,99 & 0,99 & 0,99 & 0,99 \\
\hline & $r_{g g d}$ & 0,98 & 0,98 & 0,98 & 0,98 \\
\hline & $\hat{x}$ & 12,58 & 14,94 & 18,50 & 22,49 \\
\hline \multirow{6}{*}{5} & $C V_{g}(\%)$ & 20,96 & 19,74 & 14,89 & 16,83 \\
\hline & $C V_{e}(\%)$ & 16,6 & 16,8 & 12,3 & 13,8 \\
\hline & $C V_{r}$ & 1,26 & 1,18 & 1,21 & 1,22 \\
\hline & $r_{a a}$ & 0,95 & 0,94 & 0,95 & 0,95 \\
\hline & $r_{g g d}$ & 0,96 & 0,96 & 0,96 & 0,96 \\
\hline & $\hat{x}$ & 12,19 & 15,55 & 16,18 & 20,68 \\
\hline \multirow{6}{*}{6} & $C V_{g}(\%)$ & 5,57 & 6,06 & 3,53 & 4,47 \\
\hline & $C V_{e}(\%)$ & 10,1 & 10,9 & 7,7 & 9,1 \\
\hline & $C V_{r}$ & 0,55 & 0,56 & 0,46 & 0,49 \\
\hline & $r_{a a}$ & 0,80 & 0,81 & 0,75 & 0,77 \\
\hline & $r_{g g d}$ & 0,89 & 0,92 & 0,75 & 0,82 \\
\hline & $\hat{x}$ & 13,26 & 16,22 & 18,22 & 25,17 \\
\hline \multirow{6}{*}{7} & $C V_{g}(\%)$ & 17,21 & 22,68 & 10,88 & 15,92 \\
\hline & $C V_{e}(\%)$ & 10,8 & 12,6 & 6,9 & 9,0 \\
\hline & $C V_{r}$ & 1,60 & 1,80 & 1,58 & 1,76 \\
\hline & $r_{a a}$ & 0,97 & 0,97 & 0,97 & 0,97 \\
\hline & $r_{g g d}$ & 0,99 & 1,00 & 0,99 & 1,00 \\
\hline & $\hat{x}$ & 9,39 & 10,78 & 12,80 & 15,82 \\
\hline
\end{tabular}

$C V_{g}(\%)$ : Coeficiente de variação genotípica; $C V_{e}(\%)$ : Coeficiente de variação residual; $C V_{r}$ : Coeficiente de variação relativa; $r_{a a}:$ Acurácia seletiva; $r_{g g d}$ : Correlação Genética dos materiais dentro de um sítio ou local; $\hat{x}$ : Média Geral.

$C V_{g}(\%)$ : genotypic coefficient of variation; $C V_{e}(\%)$ : residual variation coefficient; $C V_{r}$ : Relative Coefficient of variation; $r_{a a}$ : selective accuracy; $r_{g g d}:$ Genetic Correlation of materials within a site or location; $\hat{x}$ : general mean. 
As estimativas de $r_{g g d}$ para um único caráter, avaliado aos três e cinco anos, foram positivas e elevadas, variando de 0,66 a 1,00 para DAP e de 0,53 a 1,00 para ALT (Tabela 5), indicando grande probabilidade de o desempenho observado na idade juvenil se repetir à idade adulta. Os experimentos 2 e $5 \mathrm{com}$ E. grandis, experimento 4 com o híbrido e o $7 \mathrm{com}$ E. saligna demonstraram melhores correlações genéticas, com valores que variaram de 0,96 a 1,00. Dado que a correlação genética entre os caracteres possibilita predizer que a seleção direta baseada em uma característica irá beneficiar outras de forma indireta (FALCONER; MACKAY, 1996), quanto maior a correlação maior será a garantia do ganho indireto em função da seleção de uma delas. As médias gerais para DAP e altura foram similares entre experimentos (Tabela 5). O menor incremento médio entre DAP3 e DAP5 foi observado no experimento 7 (DAP5=10,78 $\mathrm{m}-\mathrm{DAP} 3=9,39 \mathrm{~m}$ ). No mesmo sentido, isto ocorreu para a altura (ALT5 $=15,82 \mathrm{~m}$ - ALT3 $=12,80 \mathrm{~m}$ ).

As $r_{f}$ entre as variáveis estudadas na idade de três (DAP3 e ALT3) e aos cinco anos (DAP5 e ALT5) variaram entre 0,75 e 0,98 (Tabela 6), indicando que a seleção praticada em uma dada variável influenciará positivamente na outra, bem como que a seleção praticada aos três anos terá comportamento semelhante aos cinco anos, o que implica em redução no tempo exigido em programas de melhoramento florestal (KAGEYAMA; VENCOVSKY, 1983; REZENDE et al., 1994).

Tabela 6. Estimativas de correlações fenotípicas $\left(r_{f}\right)$ entre diâmetro à altura do peito (DAP) e altura (ALT) em clones de Eucalyptus spp. aos três (DAP3, ALT3) e aos cinco (DAP5, ALT5) anos de idade. Local: Jacareí - SP e Capão Bonito - SP

Table 6. Correlations estimates phenotypic $\left(r_{f}\right)$ between characters for Eucalyptus spp. clones. for the character diameter at breast height (DBH) and height (ALT) at three (DAP3, ALT3) and five (DAP5, ALT5) years old. Location: Jacareí - SP and Capão Bonito - SP

\begin{tabular}{|c|c|c|c|c|c|c|c|c|c|}
\hline \multirow{2}{*}{$\begin{array}{l}\text { Experimento } \\
1\end{array}$} & \multicolumn{4}{|c|}{$\mathbf{r}_{f}$} & \multirow{2}{*}{$\begin{array}{l}\text { Experimento } \\
2\end{array}$} & \multicolumn{4}{|c|}{$r_{f}$} \\
\hline & DAP3 & DAP5 & ALT3 & ALT5 & & DAP3 & DAP5 & ALT3 & ALT5 \\
\hline DAP3 & - & 0,95 & 0,84 & 0,85 & DAP3 & - & 0,95 & 0,93 & 0,91 \\
\hline DAP5 & & - & 0,83 & 0,90 & DAP5 & & - & 0,90 & 0,94 \\
\hline ALT3 & & & - & 0,87 & ALT3 & & & - & 0,92 \\
\hline ALT5 & & & & - & ALT5 & & & & - \\
\hline 3 & DAP3 & DAP5 & ALT3 & ALT5 & 4 & DAP3 & DAP5 & ALT3 & $\overline{\text { ALT5 }}$ \\
\hline DAP3 & - & 0,98 & 0,93 & 0,96 & DAP3 & - & 0,98 & 0,93 & 0,96 \\
\hline DAP5 & & - & 0,89 & 0,95 & DAP5 & & - & 0,89 & 0,95 \\
\hline ALT3 & & & - & 0,94 & ALT3 & & & - & 0,94 \\
\hline ALT5 & & & & - & ALT5 & & & & - \\
\hline 5 & DAP3 & DAP5 & ALT3 & ALT5 & 6 & DAP3 & DAP5 & ALT3 & ALT5 \\
\hline DAP3 & - & 0,97 & 0,94 & 0,97 & DAP3 & - & 0,94 & 0,90 & 0,92 \\
\hline DAP5 & & - & 0,95 & 0,98 & DAP5 & & - & 0,87 & 0,94 \\
\hline ALT3 & & & - & 0,98 & ALT3 & & & - & 0,93 \\
\hline ALT5 & & & & - & ALT5 & & & & - \\
\hline 7 & DAP3 & DAP5 & ALT3 & ALT5 & & & & & \\
\hline DAP3 & - & 0,98 & 0,97 & 0,97 & & & & & \\
\hline DAP5 & & - & 0,95 & 0,98 & & & & & \\
\hline ALT3 & & & - & 0,97 & & & & & \\
\hline ALT5 & & & & - & & & & & \\
\hline
\end{tabular}

Baseado no DAP3, os experimentos que resultaram em $r_{f}$ superiores a 0,90 entre esta e as variáveis DAP5 e ALT5 foram os experimentos 2, 5 e 6 com E. grandis, experimento 4 com o híbrido e o 7 com E. saligna. Beltrame et al. (2012) observaram alto $r_{f}(0,92)$ entre DAP medidos aos três e aos sete anos de idade em híbridos de Eucalyptus spp. Para Massaro et al. (2010), $r_{f}$ variaram entre 0,72 e 0,98 entre DAP e ALT aos dois anos de idade e aos quatro e seis anos em clones de Eucalyptus spp. Moraes et al. (2014) consideraram como propícios à seleção precoce os caracteres DAP e ALT aos dois anos de idade em clones de Eucalyptus spp. dada às $r_{f}$ superiores a 0,70 com o DAP e ALT observados aos cinco anos. Estes elevados coeficientes de correlação, quando atrelados a diferenças significativas entre os grupos de clones e alto ganho genético de seleção, indicam que a seleção baseada na característica juvenil é uma estratégia viável para a identificação precoce de clones de Eucalyptus spp. com alto vigor de crescimento (BELTRAME et al., 2012). 
Pinto et al. (2014) observaram correlações entre DAP e ALT para E. urophylla, aos 3 e 6 anos, superiores a 0,91 . O experimento 1 com E. urophylla, apresentou $r_{f}$ inferior, com valores de 0,83 para ALT3xDAP5 e 0,95 para DAP3xDAP5, ainda assim podendo ser considerados aptos à seleção precoce (MORAES et al., 2014).

Correlações positivas e elevadas também foram observadas com base no coeficiente de correlação de Pearson, corroborando com as estimativas anteriores. No caso das correlações fenotípicas (Figura 1a), as estimativas variaram de 0,81 (DAP5 x ALT3) a 0,92 (DAP3xDAP5), todas elas estatisticamente significativas. Para correlações genéticas (Figura 1b), as estimativas foram mais elevadas e variaram de 0,99 (ALT5 x demais caracteres) a 1,00 (DAP3 x DAP5; DAP3 x ALT3; DAP5 x ALT3), sendo, também, estatisticamente significativas.
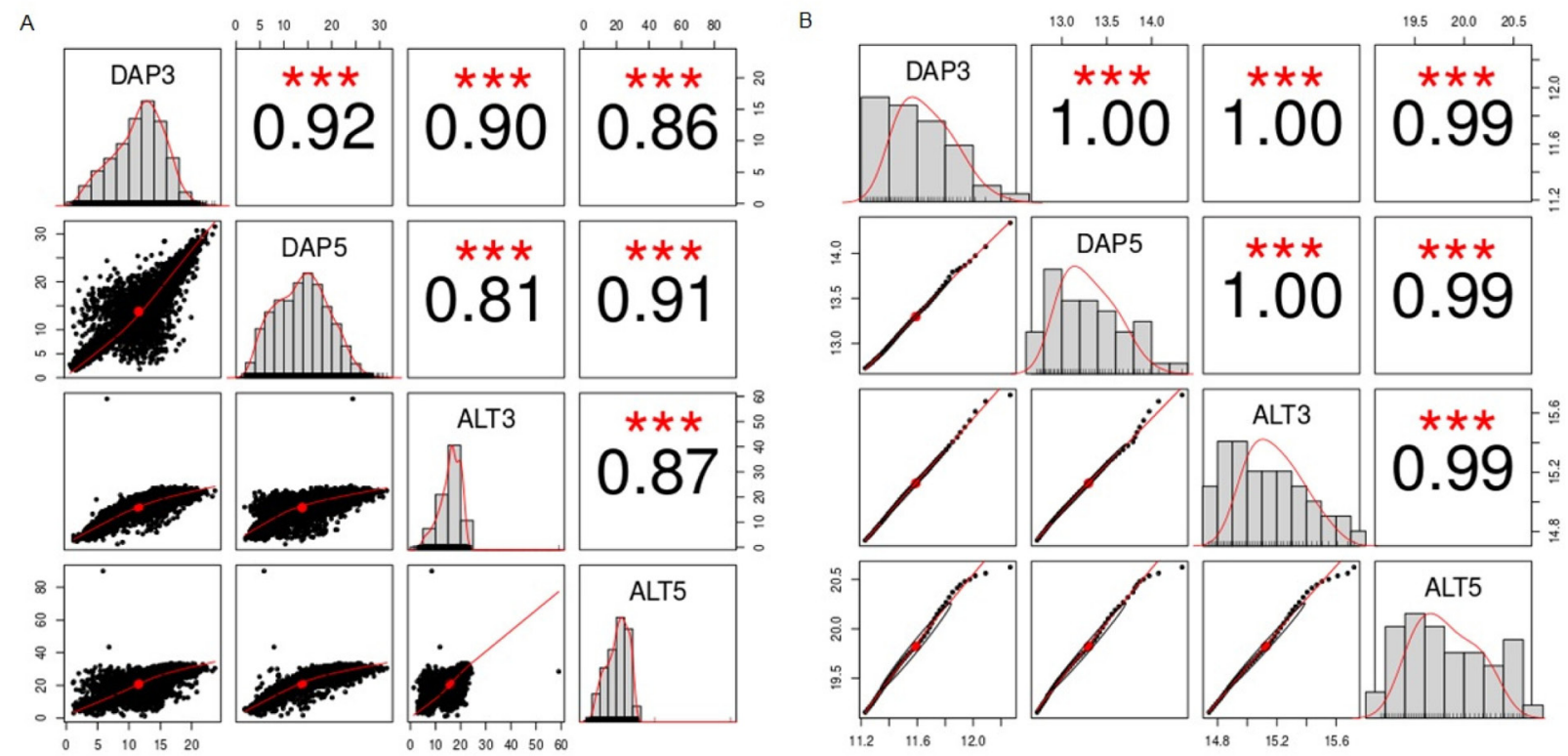

Figura 1. Estimativas de correlações fenotípicas (A) e de correlações genéticas (B), via coeficiente de correlação de Pearson, entre os caracteres diâmetro à altura do peito (DAP) e altura (ALT) em clones de Eucalyptus spp. aos três (DAP3 e ALT3) e aos cinco (DAP5 e ALT5) anos de idade. Locais: Jacareí - SP e Capão Bonito - SP. *** Significativo a $1 \%$ de nível de significância.

Figure 1. Estimates of phenotypic correlations $(A)$ and genetic correlations, by Pearson correlation coefficient, between character diameter at breast height (DBH) and height (ALT) at three (DAP3, ALT3) and five (DAP5, ALT5) years old for Eucalyptus spp. clones. Location: Jacareí - SP and Capão Bonito - SP. *** Significate at $1 \%$ of significance level.

\section{CONCLUSÕES}

Para todos os materiais genéticos avaliados, os elevados coeficientes de correlação fenotípica entre os caracteres DAP e ALT na idade de três anos e cinco anos indicam que o comportamento dos clones na fase adulta é semelhante ao observado em idades mais juvenis.

Os parâmetros genéticos estudados mostraram que a seleção de materiais é estratégia recomendada dada a alta magnitude dos mesmos.

A seleção precoce aos três anos de idade para os clones em estudo é viável e, portanto, gerará ganhos expressivos no programa de melhoramento florestal em questão.

\section{REFERÊNCIAS BIBLIOGRÁFICAS}

BELTRAME, R.; BISOGNIN, D.A.; MATTOS, B.D.; CARGNELUTTI FILHO, A.; HASELEIN, C.R.; GATTO, D.A.; SANTOS, G.A. Desempenho silvicultural e seleção precoce de clones de híbridos de eucalipto. Pesquisa Agropecuária Brasileira, Brasília, v. 47, n. 6, p. 791-796, 2012.

CRUZ, C. D.; REGAZZI, A. J.; CARNEIRO, P. C. S. Modelos biométricos aplicados ao melhoramento genético. Viçosa: UFV, 2004. v.1, 480 p. 
Queiroz et al. - Estimativas de parâmetros genéticos para a seleção precoce em clones de Eucalyptus spp.

DEAN, C.A; COTTERILL, P. P. BURDON, R. D. Early selection of radiata pine - Trends over time in additive and dominance genetic variances and covariances of growth traits. Silvae Genetica, Frankfurt, v.55, n. 4-5, p. 182-191, 2006.

FALCONER, D. S.; MACKAY, T. F. C. Introduction to Quantitative Genetics. 4. Ed. Harlow, Essex: Longmans Green, 1996, 464 p.

FERREIRA, A. R. Análise genética e seleção em testes dialélicos de Pinus taeda L. 2005. 220 p. Tese (Doutorado em Ciências Florestais) - Universidade Federal do Paraná, Curitiba, 2005.

FLORES, T. B.; ALVARES, C. A.; SOUZA, V. C.; STAPE, J. L. Eucalyptus no Brasil: Zoneamento Climático e Guia para identificação. Piracicaba, IPEF, 448p.

GARCIA, C. H.; NOGUEIRA, M. C. S. Utilização da metodologia REML/BLUP na seleção de clones de eucalipto. Scientia Forestalis, Piracicaba, v. 68, p. 107-112, 2005.

IBÁ - INDÚSTRIA BRASILEIRA DE ÁRVORES. Relatório Anual 2016: ano base 2015. São Paulo: IBÁ, 2016. $100 \mathrm{p}$.

IBÁ - INDÚSTRIA BRASILEIRA DE ÁRVORES. Relatório Anual 2015: ano base 2014. São Paulo: IBÁ, 2015. $80 \mathrm{p}$.

KAGEYAMA, P. Y.; VENCOVSKY, R. Variação genética em progênies de uma população de Eucalyptus grandis (Hill) Maiden. Revista IPEF, Piracicaba, n. 24, p. 9-26, 1983.

MARQUES JÚNIOR, O. G. Estimativas dos parâmetros genéticos e fenotípicos e avaliação da eficiência da seleção precoce em Eucalyptus cloeziana F. Muell. 1995. 69 p. Dissertação (Mestrado) - Genética e Melhoramento de Plantas, Universidade Federal de Lavras, Lavras, 1995.

MASSARO, R.A. M.; BONINE, C.A.V.; SCARPINATI, E.A.; PAULA, R.C. Viabilidade de aplicação da seleção precoce em testes clonais de Eucalyptus spp. Ciência Florestal, Santa Maria, v. 20, n. 4, p. 597- 609, 2010.

MORAES, C. B.; FREITAS, T. C. M.; PIERONI, G. B.; RESENDE, M. D. V.; ZIMBACK, L.; MORI, E.S. Estimativas de parâmetros genéticos para seleção precoce de clones de Eucalyptus para região com ocorrência de geadas. Scientia Forestalis, Piracicaba, v. 42, n. 102, p. 219-227, 2014.

PATTERSON, H. D.; THOMPSON, R. Recovery of inter-block information when block sizes are unequal. Biometrika, Londres, v. 58, p. 545-554, 1971.

PIMENTEL-GOMES, F; GARCIA, C. H. Estatística aplicada a experimentos agronômicos e florestais: exposição com exemplos e orientações para uso de aplicativos. Piracicaba: FEALQ, 2002. 309 p.

PINTO, D. S.; RESENDE, R. T.; MESQUITA, A. G. G; ROSADO, A. M.; CRUZ, C. D. Seleção precoce para características de crescimento em testes clonais de Eucalyptus urophylla. Scientia Forestalis, Piracicaba, v. 42, n. 102, p.251-257, 2014.

R CORE TEAM. R: A language and environment for statistical computing. Vienna, Áustria: R Foundation for Statistical Computing, 2016. Disponível em: < http://R.project.org/>. Acesso em: 17 out. 2016.

RESENDE, M. D. V. Matemática e estatística na análise de experimentos e no melhoramento genético. Colombo: Embrapa Florestas, 2007. 362 p.

RESENDE, M. D. V. Software SELEGEN - REML/BLUP. Colombor: Embrapa Florestas, $2002 \mathrm{a}$. 
RESENDE, M. D. V. Genética biométrica e estatística no melhoramento de plantas perenes. Brasília: Embrapa Informação Tecnológica, 2002b. 975 p.

RESENDE, M. D. V. Delineamento de experimentos de seleção para maximização da acurácia seletiva e do progresso genético. Revista Árvore, Viçosa, v. 19, n. 4, p. 479-500, 1995.

RESENDE, M. D. V.; DUARTE, J. B. Precisão e controle de qualidade em experimentos de avaliação de cultivares! Pesquisa Agropecuária Tropical, Goiânia, v. 37, n. 3, p. 182-194, 2007.

REVELLE, W. Psych: Procedures for Personality and Psychological Research: Version = 1.6.9. Evanston, Illinois: Northwestern University, 2016. Disponível em: < https://CRAN.R-project.org/package=psych >. Acesso em 14 abr. 2016.

REZENDE, G. D. S. P.; BerTOlUCCI, F. L. G.; RAMAlHO, M. A. P. Eficiência da seleção precoce na recomendação de clones de eucalipto avaliados no norte do Espírito Santo e sul da Bahia. Cerne, Lavras, v. 1, n. 1, p. 4550, 1994.

ROCHA, M. G B; PIRES, I. E.; XAVIER, A.; CRUZ, C. D.; ROCHA, R. B. Avaliação genética de progênies de meio-irmãos de Eucalyptus urophylla utilizando os procedimentos REML/ BLUP e E(QM). Ciência Florestal. Santa Maria. v. 16, n. 4, p. 369-379, 2006.

VENCOVSKY, R. Herança quantitativa. In PATERNIANI, E. (Coord). Melhoramento de milho no Brasil. Piracicaba: Fundação Cargil, 1978. p. 122.- 201.

ZIMBACK, L.; MORI, E. S.; BRIZOLLA, T. F.; CHAVES, R. Correlações entre caracteres silviculturais durante o crescimento de Eucalyptus grandis Hill ex Maiden. Revista do Instituto Florestal, São Paulo, v. 23, n. 1, p. $57-67,2011$.

Recebido em 22/11/2016

Aceito para publicação em 27/04/2017

Sci. For., Piracicaba, v. 45, n. 115, p. 507-517, set. 2017 DOI: dx.doi.org/10.18671/scifor.v45n115.08 
